\title{
LA OFERTA NATIVA DE PODCAST EN LA RADIO COMERCIAL ESPAÑOLA: CONTENIDOS, GÉNEROS Y TENDENCIAS
}

\section{The Native Podcast's Offer in the Spanish Commercial Radio: Contents, Genres and Trends}

Dra. M. ${ }^{a}$ de la Peña Mónica PÉREZ-ALAEJOS

Profesora Contratada Doctora, Universidad de Salamanca

E-mail: alaejos@usal.es

(i) https://orcid.org/0000-0001-9022-9922

Dr. Luis Miguel PEDRERO-ESTEBAN

Catedrático, Universidad Pontificia de Salamanca

E-mail: 1mpedreroes@,upsa.es

(D) http://orcid.org/0000-0003-4949-2360

Dr. Asier LEOZ-AIZPURU

Profesor Contratado Doctor, Universidad de Deusto

E-mail: asier.leoz@deusto.es

https://orcid.org/0000-0002-2949-6009

\begin{abstract}
RESUMEN
Próxima a cumplir su primer siglo e inmersa en un entorno de convergencia y desintermediación, la radio comienza a diseñar modelos productivos transversales que, además de cubrir la transmisión en antena e Internet, se orientan al consumo asincrónico del audio sobre la premisa inherente al actual ecosistema: cualquier contenido ha de estar disponible en cualquier momento y para cualquier dispositivo. El afianzamiento del podcast ha crecido de forma exponencial hasta crear un escenario de consumo sonoro alternativo al de la radio analógica. En este contexto, los operadores tradicionales asumen la producción de contenidos para su difusión exclusiva online bajo criterios de segmentación menos restringidos por el mercado publicitario hertziano. Este trabajo identifica la oferta de audio radiofónico digital bajo la hipótesis de que su construcción pretende introducir la marca radiofónica en las nuevas pantallas antes que experimentar con otras narrativas o atender a diferentes audiencias. Para ello analiza el catálogo de podcasts nativos de las tres grandes cadenas generalistas privadas de cobertura estatal (SER, COPE, Onda Cero) durante la temporada 2017/18. El objetivo es reconocer, clasificar y valorar sus contenidos, géneros y tendencias en un escenario cuya evolución resultará determinante para consolidar la pervivencia de la producción radiofónica.
\end{abstract}

Palabras clave: Podcast; Radio; Audio; Consumo digital; Narrativas.

\section{ABSTRACT}

Close to its first centenary and immersed in a convergence environment, radio begins to design transversal productive models oriented towards the personal consumption of audio according to the main premise in the current eco- 


\begin{abstract}
system: all contents must be available at all times and for all devices. The podcast acceptance has exponentially grown to create a sound consumption scenario destined to replace to the analogue radio. In this context, traditional operators assume the production of content designed for exclusive online dissemination under segmentation criteria less restricted by the Hertzian market. This work identifies the digital audio offer under the hypothesis that its construction still prefers to introduce the radio brand on new screens rather than experimenting with other narratives or providing services to different audiences. According to this, it analyses the catalogue of native podcasts of the three large private generalist chains of state coverage (SER, COPE, Onda Cero) during the 2017/18 season. The objective is to recognize, classify and assess their themes, genres and strategies in a scenario whose evolution will be decisive to consolidate the survival of radio production.
\end{abstract}

Key words: Podcast; Radio; Audio; Digital Consumption; Storytelling.

\title{
1. LA TRANSFORMACIÓN DIGITAL DE LA RADIO
}

La aceleración en la migración del sistema mediático al entorno digital y el empleo masivo de los smartphones como dispositivos dominantes de acceso a la información y el entretenimiento han impuesto una progresiva e irreversible transformación sobre el funcionamiento de las industrias culturales. El cambio del escenario analógico a un paisaje audiovisual de conectividad, ubicuidad, atemporalidad e interactividad (Siemens, 2008) está redefiniendo las tradicionales rutinas de producción, distribución y comercialización de contenidos televisivos, radiofónicos, musicales y cinematográficos, aunque con ritmos dispares y perspectivas de explotación aún inciertas debido a la convivencia de dos públicos diferenciados: quienes asumen activamente las emergentes herramientas tecnológicas -en especial los perfiles más jóvenes de población- y los que todavía siguen utilizando soluciones en declive o en vías de extinción (Díaz Nosty, 2017). Aunque la consolidación de otros soportes, canales y plataformas ha incrementado el tiempo de exposición de ambos grupos de audiencias, se aprecian dietas mediáticoculturales distintas según el segmento generacional de procedencia que vaticinan futuros efectos disruptivos de las nuevas vías de comunicación. De ahí la inicial conveniencia -ya exigencia- de cada industria para adaptar e incluso reinventar la concepción, el diseño, las narrativas y hasta los modelos de negocio sobre los que sostuvieron su actividad antes de Internet (Pedrero y Pérez-Alaejos, 2018).

Próxima a cumplir su primer siglo la radio se halla sumida en pleno proceso de transición a un paradigma insólito en el que ya no mantiene el monopolio en la creación y distribución del audio. Sus esfuerzos se vuelcan ahora en identificar y explorar la viabilidad comercial que alcanzan en el ciberespacio las plataformas automatizadas, el podcasting y la hibridación de medios sonoros y visuales. Sin embargo, y en contraposición a otras industrias, muchas de las iniciativas, dinámicas y experiencias probadas en cada continente, país o región evidencian la falta de estrategias más o menos pautadas por sus agentes para consolidar en el nuevo escenario la idiosincrasia del medio de comunicación con mayor prestigio e impronta: pese al descrédito en el que han incurrido la televisión, los periódicos y revistas o las rutilantes redes sociales, la radio sostiene elevados niveles de credibilidad en toda Europa (European Broadcasting Union, 2017) y sigue acreditando su beneficio social en situaciones donde aún no llegan las prestaciones de la Red.

Son muchos los autores que han identificado la lentitud en este proceso como una demostración de la renuencia de la industria para asumir el cambio: «Acometer la transición digital progresiva entendida de manera holística podría constituir el revulsivo idóneo para transformar la radio y adaptar su estructura empresarial a los retos de producción crossmedia y, a su vez, cambiar la mentalidad de los productores radiofónicos anclados en los contenidos monomedia» (Franquet, 2017). Si bien el sector es consciente de que la difusión de ondas hertzianas a través de transistores tiene fecha de caducidad y asume que debe replantear sus estrategias con el fin de evitar el indeseado trasvase de las audiencias a 
plataformas más ágiles, creativas, entretenidas y abiertas (López Vidales et al., 2014), a menudo las fórmulas ensayadas han parecido responder más a la exploración que al convencimiento.

Con todo, el asentamiento de hábitos ligados al ocio digital y el disfrute que de él hacen las nuevas generaciones de usuarios han terminado por inducir a los radiodifusores a la puesta en marcha de modelos productivos transversales con los que cubrir la retransmisión en directo (antena e Internet) y, sobre todo, facilitar el consumo asincrónico y personalizado del audio (Videla-Rodríguez y PiñeiroOtero, 2013; Orrantia, 2018). La consolidación del consumo audiovisual a demanda popularizada por las plataformas de música y vídeo está desdibujando el alcance e incluso la lógica de las programaciones lineales de los operadores clásicos de televisión y radio, si bien la relevancia del medio sonoro entre el público más joven aún resulta menor debido al desinterés con el que por distintas razones la radio ha tratado históricamente a niños y adolescentes (Catalina et al., 2017). De ahí la trascendencia de las nuevas apuestas de difusión digital, especialmente el podcast, cuya evolución se relata a continuación para situar el contexto de producción y comercialización sobre el que la industria tradicional está articulando sus creaciones sonoras a la carta.

\section{EL PODCASTING: EVOLUCIÓN Y TENDENCIAS}

Si hay un medio históricamente acostumbrado a resistir en condiciones desfavorables es la radio: lo ha hecho desde que en los años 30 del siglo pasado la prensa la etiquetase como seria competidora y forzó a las agencias de noticias a elegir entre ambos medios (Tenorio, 2008) hasta que, décadas más tarde, la llegada de la televisión le auguró un abrupto final, seducidos anunciantes y público por un medio nuevo y más potente que conquistó los hogares a través de los ojos (Casas Santasusana, 1954). En España la radio adquirió tras la dictadura un carácter netamente informativo que la condujo a un férreo modelo de programación marcado por el inmovilismo (Balsebre et al., 2006). Y hoy la revolución social y tecnológica, que ha asentado un entorno digitalizado y de participación, invita a combatir ese inmovilismo y a reinventarse una vez más, a repensar el modelo radiofónico dirigido a un oyente que ahora ya es usuario y poco tiene que ver con el de décadas anteriores.

Una de las claves de esta gran transformación es el podcast. Cuando el periodista Ben Hammersley acuñó ese término en el diario británico The Guardian (2004) planteaba una perspectiva de aficionados en la elaboración y difusión de mensajes radiofónicos hoy superada. El podcasting resulta un elemento imprescindible en la radio profesional e incluso fuera de este ámbito: en nuestro país los 24 millones de descargas de los programas alojados en la plataforma Podium Podcast solo dos años después de su lanzamiento (2016) o los datos referentes a los podcasts más descargados evidencian la importancia de un formato crucial en el ecosistema radiofónico contemporáneo.

La revolución audible de la que Hammersley se hacía eco en su artículo tenía lugar tres años después de que Apple pusiera en circulación su revolucionario reproductor de música en MP3, el iPod. En realidad, la escucha portátil del sonido grabado se había materializado mucho tiempo atrás: en los 80 existía el walkman, que reproducía cassettes, y después llegó el discman, capaz de hacer sonar CDs. Pero el iPod de Apple, que su creador Steve Jobs presentó recalcando la capacidad de «albergar la discografía completa de una persona en el bolsillo de su pantalón» (Jobs, 2001), dejaba muy atrás la capacidad de aquellos equipos. El iPod permitía escuchar archivos de sonido comprimidos mediante la tecnología que el instituto de investigación alemán Fraunhofer-Gesellschaft había desarrollado desde 1993 (Witt, 2016). Fue un rotundo éxito en una época en la que el diseño de compresores de sonido, y paralelamente de Internet, puso en jaque a la industria discográfica al permitir al usuario hacer copias perfectas de sus discos (o de los que no le pertenecían) y compartirlos en la Red a través de la tecnología P2P. Es en ese contexto cuando The Guardian une los términos pod (cápsula) y broadcast (transmi- 
sión), dando lugar al concepto de podcast. Sin pretenderlo, Apple obtuvo gran ventaja competitiva al generalizarse el uso de la palabra, pues sugería una cierta exclusividad o relación directa con la compañía que en realidad no existía (Berry, 2006).

«El podcasting a nivel tecnológico es la unión de dos tecnologías ya conocidas anteriormente como son el MP3 y el RSS. El RSS ha facilitado la suscripción a una fuente que automatiza la descarga de los archivos MP3 generados previamente» (Gallego, 2010, p. 34). La sindicación de contenidos dio lugar a una relación diferente entre el emisor - portal web, blog o prensa online- y el receptor, que no precisa visitar la fuente para descubrir si hay nuevos contenidos y consumirlos (Nafría, 2008). El podcast rompió así con la relación temporal entre la emisión y la recepción, igual que el hipertexto lo había hecho antes con la lectura secuencial de textos escritos.

En relación con esta tecnología es significativa la reflexión de Josep M. ${ }^{a}$ Martí en el año 2000: un año antes del lanzamiento del iPod auguraba a la radio transformaciones técnicas más grandes que las causadas por la llegada de la FM en 1979. Aún reconociendo que la relación de radio e Internet estaba en una fase de desarrollo inicial, acertó al afirmar que la digitalización iba a provocar una segmentación de formatos y audiencias según sus gustos, lo que impulsaría el desarrollo de formatos a la carta o de audio on demand (Pedrero, 2000, p. 244). Por tanto, no solo se iba a permitir al oyente acceder a los contenidos como y cuando lo deseara, sino que las propias emisoras tendrían que pensar también que ese oyente será cada vez más exigente y querrá disfrutar de contenidos que tal vez no emitan las cadenas que escucha, por lo que buscará en otros lugares o, con una modesta inversión económica de por medio, los podrá elaborar por su cuenta y distribuir después.

Algunos expertos señalaron en 2006 que el valor añadido del medio podía estar en Internet, canal en el que se interrelacionan digitalización, convergencia e interactividad, porque da lugar a múltiples opciones para cualquier emisora convencional y también al nacimiento de nuevos operadores, favoreciendo la experimentación de otras modalidades de información y expresión, incluida la publicitaria, que van más allá del sonido radiofónico, y generando nuevas formas de consumo y de relación con el medio (Balsebre et al., 2006). La era de Internet posibilitaba un escenario donde la radio podía explotar aún más que antes sus formidables capacidades de enganche y fidelización del oyente.

Sin embargo, el proceso ha sido menos rápido de lo que prometía. Aunque Berry advirtió que el podcast, además de sintetizar la convergencia de sonido, Internet y dispositivos portables, suponía una tecnología disruptiva que forzaría al negocio radiofónico a reconsiderar prácticas establecidas e ideas preconcebidas sobre audiencias, consumo, producción y distribución (Berry, 2006), los efectos de tal impulso no se percibieron de inmediato. A finales de esa misma década las emisoras aún mantenían el control sobre los contenidos, pero no habían desarrollado una oferta específica para Internet ni generado un nuevo modelo de negocio fundamentado en el consumo en la Red. Por tanto, el esquema unidireccional seguía siendo el mismo que en la difusión hertziana.

Al finalizar la primera década de este siglo diversos trabajos de investigación sobre el modelo radiofónico español apuntaban a su agotamiento, causado por el afán de copiar formatos y contenidos en las ondas para volcarlos en la Red sin repensar el medio ni buscar un nuevo modelo de negocio. En la radio española se echó en falta una reflexión sobre el camino que debía tomar con relación al podcast. lo mismo que sucedió antes de la crisis de las puntocom, el uso de Internet por parte de los operadores no respondía a una estrategia clara de diversificación y distribución de contenidos, sino al deseo de no quedar por detrás de la competencia. Las posibilidades de un mercado segmentado basado en Internet y de una economía de larga cola (Anderson, 2004) parecían aún por explotar.

Los investigadores Pérez-Alaejos y López Merayo (2013) aportan una lectura reveladora sobre las cadenas generalistas españolas en relación con el podcast. concluyen que la mayoría utiliza los portales 
web y el audio a la carta como base de su programación en la Red y reclamo para una audiencia que quiere consumir contenidos concretos sin estar sujeta a los horarios de emisión en las ondas; sin embargo, pese a poner sus audios al alcance del usuario, no aprovechan Internet para ofrecer contenidos diferentes y novedosos sin cabida en las ondas. Ponen además el foco en la publicidad, revelando que la que se encuentra en los podcasts equivale a la que contenían los propios programas en el momento de emisión y en el mismo orden secuencial, una prueba más de la falta de aprovechamiento de las posibilidades del podcast respecto a la radio tradicional.

En 2014 el periodista Javier Gallego deja la Cadena SER por no compartir objetivos ni sintonía con la cadena. Su programa Carne cruda -Premio Ondas 2012- desaparece entonces de la parrilla, pero él continúa emitiéndolo a través de su sitio web (www.carnecruda.es) hasta que el periódico nativo digital eldiario.es lo aloja en su medio, algo impensable no demasiado tiempo atrás. Se constata así cómo un programa nacido al abrigo de una cadena tradicional podía salir adelante sin su respaldo: la revolución que supone el podcasting en la profesión radiofónica comporta una mayor libertad, sí, pero no solo para el usuario, como este y otros casos que sucedieron después (por ejemplo, el de Iker Jiménez en Radioset, el portal de audio de Mediaset) pusieron de manifiesto.

El profesor italiano Tiziano Bonini identifica algunos hitos que permiten deslindar la primera era del podcasting de la actual. Por encima de todos destaca uno: 2012, año en el que algunos de los más famosos podcasts de la radio pública norteamericana NPR pasaron a ser independientes y comenzaron a financiarse vía crowdfunding desde plataformas como Kickstarter (Bonini, 2015). Un año después, al tiempo que percibe un lento pero constante descenso de sus oyentes en la FM, la BBC produce 263 podcasts, casi un tercio de su producción de programas. Es el resultado de un viraje que se asienta en grandes corporaciones como la citada BBC o la NPR norteamericana desde 2006.

Como sostiene Bonini, la financiación resultó clave para poder hablar de una nueva fase en este entorno. Plataformas de micro-mecenazgo como la citada Kickstarter o Indie Go Go, sumadas a redes sociales basadas en el sonido como SoundCloud, Spreaker y Stitcher, recuperaron el «espíritu DIY» (hazlo tú mismo) que impulsó a la cultura punk en los años 80 a crear productos culturales al margen del mainstream o mercado de masas. El matiz es que, en cuanto a calidad sonora y de realización, no existen sustanciales diferencias entre los productos elaborados por corporaciones de comunicación y por creadores sin relación alguna con una compañía.

Muestra de ello son los datos del principal repositorio de podcast de España: iVoox (Tabla 1). En abril de 2018, Mediaset, la Cadena SER y Onda Cero se repartían los primeros puestos entre los audios más descargados en nuestro país. Pero no solo había grandes grupos de comunicación: entre los veinte primeros se hallan programas y creadores de contenidos ajenos a ellos, como Santiago Camacho (DEX) al frente de Días extraños, o La escóbula de la brújula. Se observa cómo el escenario actual permite que quien crea un programa atractivo o diferencial y lo distribuye a través de plataformas de alojamiento y promoción -junto a iVoox y Podium, se han asentado en España Cuonda, Spain Media Radio, Nación Podcast, Emil Car, AV Podcast o DCast, entre otras- puede alcanzar un impacto de audiencia y publicidad equiparable al de las tradicionales cadenas hertzianas, pese a que las métricas de unos y otros todavía no permiten una clara equiparación. 
Tabla 1: Top 20 podcasts más descargados en iVoox (abril 2018)

\begin{tabular}{|l|l|l|l|}
\hline N. $^{\circ}$ & PROGRAMA & PRESENTADOR/ES & OPERADOR \\
\hline 1 & Cuarto milenio & Iker Jiménez & Mediaset \\
\hline 2 & Nadie sabe nada & A. Buenafuente y B. Romero & Cadena SER \\
\hline 3 & Oh my LOL & David Broncano & Cadena SER \\
\hline 4 & La rosa de los vientos & Bruno Cardeñosa & Onda Cero \\
\hline 5 & Documentales sonoros & desconocido & Discovery Channel \\
\hline 6 & Días extraños & Santiago Camacho & - \\
\hline 7 & Universo Iker (Oficial) & Iker Jiménez & Radioset \\
\hline 8 & Misterios & desconocido & - \\
\hline 9 & Espacio en blanco & Miguel Blanco & RNE \\
\hline 10 & SER Historia & Nacho Ares & - \\
\hline 11 & El partidazo de la COPE & Juanma Castaño & COPE \\
\hline 12 & La escóbula de la brújula & J. Callejo, D. Sentinella... & Podium Podcast \\
\hline 13 & Es la mañana de Federico & Federico Jiménez Losantos & EsRadio \\
\hline 14 & Momentos & Luis Rodríguez & COPE \\
\hline 15 & Mundo insólito radio & desconocido & - \\
\hline 16 & La ContraCrónica & Fernando Díaz Villanueva & - \\
\hline 17 & El transistor & José Ramón de la Morena & Onda Cero \\
\hline 18 & Julia en la Onda & Julia Otero & Onda Cero \\
\hline 19 & Luces en la noche & desconocido & - \\
\hline 20 & Antena historia & desconocido & - \\
\hline
\end{tabular}

Fuente: elaboración propia a partir de datos de iVoox

La llegada de Podium Podcast marca el inicio del podcasting profesional en España, al romper con el amateurismo que impregnaba este formato. Según Vicente Jiménez, director de PRISA Radio cuando se lanzó la plataforma, el proyecto surgió ante la obligación de atender a un oyente digital que dejó de ser sujeto pasivo y quiere consumir radio en cualquier lugar y desde cualquier dispositivo (Morla, 2016): «No es radio. Pero siendo audio, mantiene el lazo familiar con la radio», apuntó durante la presentación Antonio Hernández Rodicio, entonces director de la Cadena SER. Su éxito nos lleva a recuperar la siguiente reflexión sobre el poder del usuario, que no mero oyente, de radio.

Ahora la audiencia es activa en la praxis y selecciona, programa, remezcla, produce e interactúa. La clave del futuro de la comunicación pasa por entender y encauzar todas las inquietudes de la nueva audiencia activa. (Gallego, 2010, p. 138).

El podcast es, por tanto, la gran salida para el cada vez más determinante poder de los usuarios en su relación con los programas de radio. Definitivamente desligados de la rigidez de la emisión tradicional, viven la experiencia de escucha de una manera nueva y abierta a posibilidades. Doce años después de que la revista Time nombrase al 'usuario' como 'personaje del año' (2006), la radio ofrece una enorme gama de posibilidades de crecimiento y se obliga nuevamente a repensar su modelo sin renunciar por ello al espíritu que la convierte en un medio especial y sugerente.

\section{LA OFERTA NATIVA DE PODCAST EN LA RADIO ESPAÑOLA}

Aunque la proliferación y diversificación de contenidos de audio distribuidos a través de Internet ha ampliado las acepciones que en su origen designaba el término podcast, se puede considerar que en la actualidad conviven dos grandes variantes: el podcast de programa, básicamente contenidos de programas fragmentados y subidos a la web por las emisoras para prolongar su vida más allá de la antena; y el podcast nativo digital, contenidos que nacen y se desarrollan en un entorno exclusivamente online 
sin difusión previa en antena tradicional. Este último modelo vive un gran momento en Estados Unidos y factura más que la radio convencional gracias a series de ficción, periodísticas o de entretenimiento con una gran capacidad de monetización vía suscripción.

En España se han puesto en marcha en los últimos años proyectos de producción y agregación de podcasts desde operadores externos a la industria tradicional e incluso desde la propia radio, como la ya reseñada plataforma de PRISA Podium Podcast, con una oferta exclusiva que intenta responder a las necesidades, gustos e intereses del oyente (Moreno, 2017) aprovechando parte del archivo histórico de la Cadena SER (Rodríguez Pallarés, 2016). Este doble proceso, de adaptación (legacy media) y de especificidad (medios nativos digitales), permite hablar de una nueva narrativa digital cuyos elementos definitorios y distintivos son la hipertextualidad, la interactividad y las posibilidades multimedia, y que se manifiesta a través de formas de producir contenido crossmedia para nuevas formas de escucha mediante formatos que responden a estructuras diferentes a las tradicionales y aprovechan los contenidos generados por los usuarios y sus posibilidades para encauzar procesos cada vez más participativos, personalizados y envolventes.

La llegada de las tecnologías digitales ha impulsado iniciativas sonoras emergentes cuyo potencial innovador no está siendo siempre suficientemente aprovechado por las cadenas de radio en España. Ya en 2013 algunos autores concluían que «la radio española no exprime Internet para ofrecer contenidos novedosos y alternativos sin cabida en las ondas. Los podcasts generados son sencillamente copias idénticas de los programas analógicos, que ni siquiera guardan una estructura fija de fragmentación en la mayor parte de los casos. Están concebidos como el soporte en el que emitir de otra forma los mismos contenidos para ser consumidos a la carta, pero sin el diseño de una nueva narrativa específica» (Pérez Alaejos y López Merayo, 2013, p. 199).

Cinco años después, el presente trabajo pretende identificar, reconocer y valorar las temáticas, géneros y estrategias -duración, caducidad o renovación- de la oferta sonora nativa de las tres cadenas comerciales españolas de mayor penetración y audiencia con el fin de constatar si sus apuestas por este formato revelan nuevas estrategias acordes con las demandas propias del ecosistema digital y contribuyen de ese modo a que la industria radiofónica se sitúe en una posición competitiva frente a las crecientes y cada vez más diversificadas propuestas de ocio y entretenimiento.

\section{Metodología}

Se ha utilizado el análisis de contenido para contabilizar los podcasts nativos digitales que los operadores comerciales de programación generalista -SER, COPE y Onda Cero- han producido durante la temporada 2017/2018, eligiéndose para el estudio los espacios disponibles en sus ventanas digitales. La muestra se justifica por el alcance de las tres cadenas tanto en términos de audiencia como de facturación publicitaria: según el Estudio General de Medios de la 2. a oleada de 2018 (junio), la Cadena SER cumplió 25 años ininterrumpidos de liderazgo de la radio española con 4.089 .000 oyentes diarios de lunes a viernes, por encima de la Cadena COPE, que sumó 2.686.000 seguidores, y de Onda Cero, con 1.939.000. Las tres superaron las cifras de Radio Nacional de España: 1.391 .000 oyentes. Para la toma de datos se ha usado como herramienta el sitio web de cada cadena y la información que en él se ofrece sobre estos contenidos. Cada uno de los podcasts producidos para consumo exclusivo digital ha sido considerado como una unidad de análisis, contabilizándose un total de 44 entre las tres cadenas analizadas. Así se ha llevado a cabo una investigación empírico-descriptiva que pretende detallar las características de las experiencias innovadoras en España, con especial foco en la radio online.

Para el cómputo de los datos se ha recurrido a una plantilla donde se han identificado distintas variables relevantes para el objeto de estudio en cada unidad de análisis. En ella se han codificado los 
contenidos producidos para el consumo online por cada cadena durante la temporada 2017/18; se ha prestado atención al contenido completo, es decir, al programa, y cada uno de ellos ha sido considerado una unidad específica. Además de registrar la cadena titular, se ha identificado el nombre del programa, el tema principal y el subtema específico para recoger mejor la esencia del espacio analizado.

El libro de códigos incluye también la duración de estos espacios y su periodicidad de aparición, catalogando el género periodístico donde se encuadran y si la producción pertenece a la propia cadena o se trata de una producción externa. La plantilla contiene, además, información sobre la presencia o no de la fórmula publicitaria del patrocinio (única sobre la que hasta el momento se ha comercializado este formato digital), la fecha más antigua en la que la cadena ofrece al usuario dicho contenido y si el podcast se ofrece completo o fragmentado. El análisis estadístico de estos datos permite ofrecer aquí de manera sistematizada los siguientes resultados.

\section{Resultados}

La investigación sobre la oferta nativa de podcasts en las principales cadenas generalistas españolas de radio comercial ha servido para componer una radiografía muy ilustrativa sobre la concepción y las expectativas que cada operador concede actualmente a este formato de audio digital. De las 44 unidades de análisis, 21 corresponden a la Cadena COPE y 15 a la Cadena SER, mientras Onda Cero ofrece en su web únicamente 8 espacios de difusión exclusiva online. La mitad de estos productos $(50 \%$ del total) se dedican a contenidos deportivos, seguidos por los culturales, que representan poco más de la cuarta parte $(27,3 \%)$. La temática social apenas alcanza un $6,8 \%$, y la educativa se queda en el $4,5 \%$. El $9,1 \%$ restante se dedica a otros temas como salud, gastronomía, especiales temáticos y ficción.

De los 22 podcasts deportivos, la mitad (11) está dedicada a deportes como el rugby, el ciclismo, el running o el tenis, mientras que 8 se centran en el fútbol y 3 en el baloncesto. De los 12 culturales, 3 están dedicados al cine y 7 son especiales musicales o temáticos: COPE produce semanalmente uno con información taurina especializada y otro sobre cocina. Programas como Geniales Cope, Latitud Cero o Generación Euskadi abordan en profundidad asuntos sociales como la discapacidad, el conflicto de los refugiados de guerra o los hábitos de consumo de los más jóvenes.

Gráfico 1: Distribución de podcasts nativos por temática

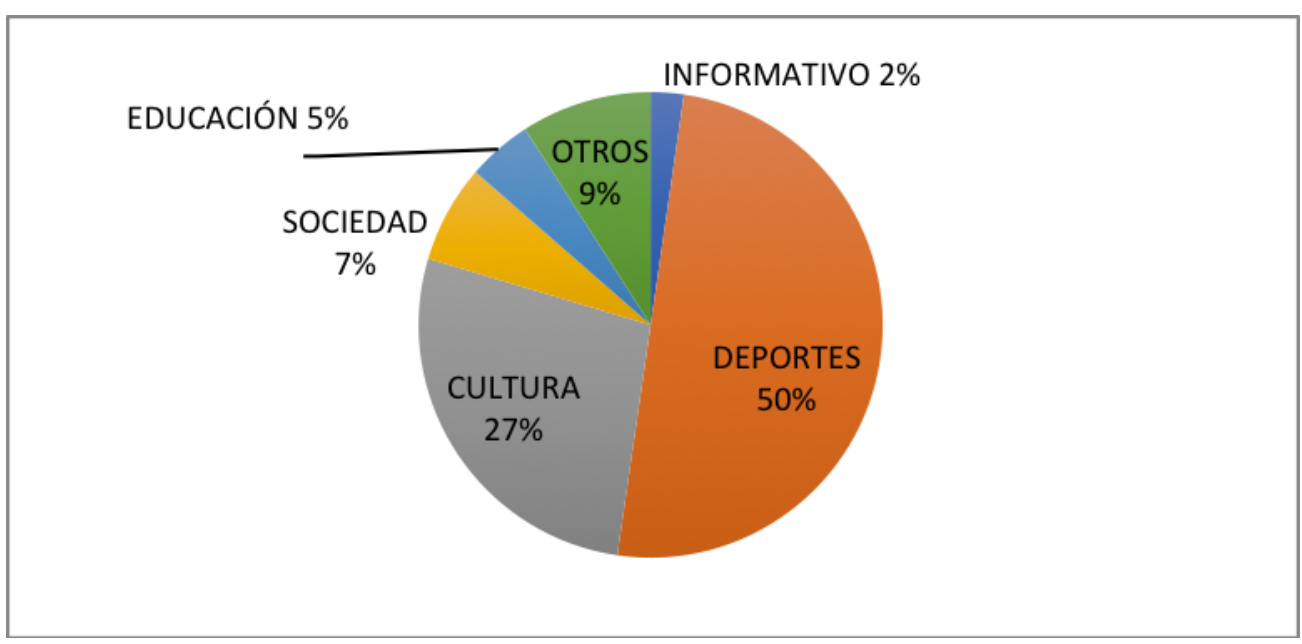

Fuente: elaboración propia 
Sobre la duración de los programas, 8 de los 44 se extienden más allá de una hora, y tan solo dos quedan por debajo de los diez minutos. Se han registrado 11 podcasts con una extensión de entre 21 y 30 minutos, y otros 10 entre 51 y 60, de modo que la mayoría (70,4\%) se sitúa en la franja de 21 a 60 minutos. En este apartado resulta relevante reseñar que el 65,9\% de los podcasts analizados tienen una periodicidad semanal, solo el $9,1 \%$ se produce cada quince días y el $22,7 \%$ lo hace sin periodicidad fija, con saltos temporales y frecuencias discontinuas. El 2,3\% de los casos analizados solo se ha producido en una ocasión, coincidiendo con algún acontecimiento informativo especial.

Gráfico 2: Periodicidad en la producción de podcasts nativos

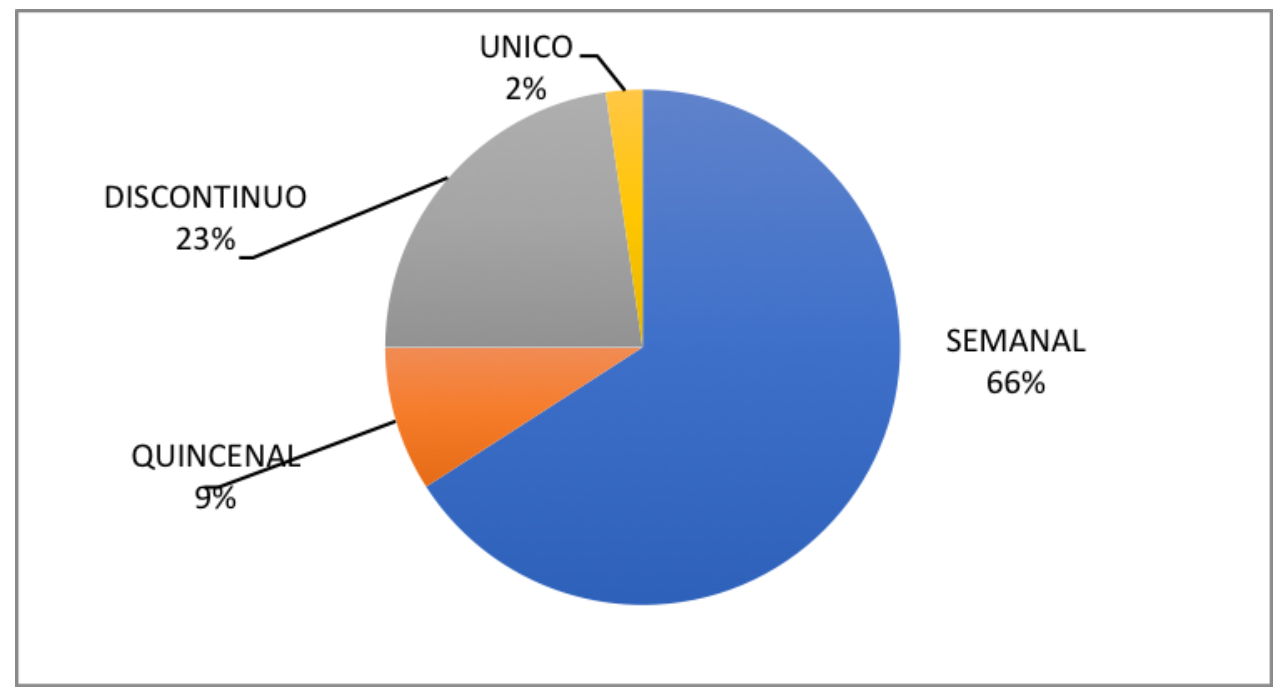

Fuente: elaboración propia

Si atendemos al género radiofónico sobre el que se articulan, cabe subrayar que el 54,5\% de los casos se corresponde con el magazine que ocupa el primer puesto a gran distancia del género informativo $(13,6 \%)$. El 9,1\% y el 6,8\% se etiquetan como reportajes y entrevistas, mientras que solo se ha registrado un caso de ficción (Sin Mi Identidad, en COPE) de los 44 analizados. El 11,4\% restante se basa en géneros categorizados como «otros» por su indefinición y/o mezcla.

Tabla 2: Géneros de podcasts por cadenas

\begin{tabular}{|l|c|c|c|c|}
\hline \multicolumn{1}{|c|}{ GENERO } & SER & COPE & ONDA CERO & TOTAL \\
\hline Magazine & 8 & 16 & 0 & 24 \\
\hline Informativo & 0 & 2 & 4 & 6 \\
\hline Reportaje & 0 & 0 & 4 & 4 \\
\hline Entrevista & 3 & 0 & 0 & 3 \\
\hline Ficción & 0 & 1 & 0 & 1 \\
\hline Otros & 4 & 1 & 0 & 5 \\
\hline
\end{tabular}

Fuente: elaboración propia

Con respecto a la comercialización de estos podcasts nativos, y aunque la mayoría van precedidos por publicidad en audio o vídeo previo a su descarga o reproducción, tan solo 4 de los 44 analizados incluyen un patrocinio dentro del contenido sonoro. Es interesante destacar también que 38 de los 44 podcasts han sido producidos por la propia cadena, 6 por emisoras asociadas a la marca, generalmente 
de ámbito local o regional, y ninguno de ellos es producido de manera externa, o al menos, no se refleja así en su guion.

Finalmente, y atendiendo a la renovación de los respectivos catálogos, solo el 29,5\% de los podcasts analizados son de nueva creación en la temporada 2017/18, ya que el 70,5\% están al alcance del usuario, como el caso de Play Futbol de la SER, desde el año 2010. COPE es la cadena que ha incorporado más novedades a su repertorio online en el último año, ya que mientras Onda Cero solo ha sumado 3 nuevas producciones, la cadena de la Conferencia Episcopal alcanzó los 9 registros.

\subsection{CADENA SER}

Durante la temporada 2017/18 la Cadena SER ofreció en su web un total de 15 programas nativos digitales, aunque solo incorporó uno nuevo con relación a cursos anteriores. Sus contenidos deportivos online sobre fútbol y baloncesto (Play fútbol y Play basket) son dos de los más antiguos en la radio comercial española, y a ellos se han sumado otros dedicados al rugby, al running o al tenis, aumentando de manera significativa su oferta especializada deportiva. Llama la atención que 8 de los 15 podcasts tienen periodicidad discontinua, mientras que los restantes se ofrecen semanalmente. Es reseñable también que algunos de sus podcasts culturales están producidos por emisoras asociadas como Radio Bilbao o Radio Mallorca, y que tan solo cuatro de los deportivos superan la hora de duración, mientras que la mayoría restante no excede los 30 minutos.

Tabla 3: Oferta de podcasts nativos en la Cadena SER

\begin{tabular}{|l|c|c|c|c|}
\hline \multicolumn{1}{|c|}{ Título } & Tema & Subtema & Duración & Periodicidad \\
\hline In Pod We Cast & Otros & Otros (cultura americana) & $00: 33^{\prime}$ & Discontinua \\
\hline Play Gastro & Otros & Otros (cocina) & $00: 26^{\prime}$ & Discontinua \\
\hline Fuego y chinchetas & Cultura & Especiales musicales & $00: 24^{\prime}$ & Discontinua \\
\hline BeOK & Otros & Salud & $00: 25^{\prime}$ & Semanal \\
\hline La Script & Cultura & Cine & $00: 29^{\prime}$ & Discontinua \\
\hline Cara A & Cultura & Especiales musicales & $00: 23^{\prime}$ & Discontinua \\
\hline Cultura Pod & Cultura & Otros (cultura musical) & $00: 13^{\prime}$ & Discontinua \\
\hline Generación Z Euskadi & Sociedad & Otros (temas actualidad) & $00: 21^{\prime}$ & Discontinua \\
\hline SER Runner & Deporte & Otros deportes & $00: 44^{\prime}$ & Semanal \\
\hline Play Futbol & Deporte & Fútbol & $01: 08^{\prime}$ & Semanal \\
\hline Play Basket & Deporte & Baloncesto & $01: 49^{\prime}$ & Semanal \\
\hline Punto, SER y Partido & Deporte & Otros deportes & $00: 27^{\prime}$ & Discontinua \\
\hline Play Segunda & Deporte & Fútbol & $01: 15^{\prime}$ & Semanal \\
\hline 100 Yardas & Deporte & Otros deportes & $01: 22^{\prime}$ & Semanal \\
\hline Play Rugby & Deporte & Otros deportes & $01: 26^{\prime}$ & Semanal \\
\hline
\end{tabular}

Fuente: elaboración propia

Como se señaló más arriba, la oferta nativa digital de la Cadena SER -la principal marca de PRISA Radio- se concentra desde junio de 2016 en la plataforma Podium Podcast, con un repertorio específico y autónomo del catálogo de la cadena -aunque de manera esporádica suenan en antena algunas de estas producciones- donde se atiende con mayor énfasis a la ficción y al entretenimiento, y cuya monetización discurre por parámetros distintos a los de los podcasts nativos analizados. El esfuerzo en la producción de contenidos exclusivos online está contribuyendo al afianzamiento de este formato sonoro, a la aparición de una competencia cada vez más amplia y exigente y, sobre todo, a la popularización del consumo de audio digital: a los veinte meses desde su lanzamiento (marzo de 2018) la plataforma había alcanzado veinte millones de descargas, un hito en la historia del podcast en España. En todo caso, la Cadena SER revela en su catálogo de contenidos de audio online su alineamiento con la actualidad, especialmente la deportiva, y la búsqueda de nichos de audiencia más segmentada que la que conforma su público de antena convencional. 


\subsection{CADENA COPE}

COPE ha resultado ser la cadena generalista que produce un mayor número de podcasts exclusivos para su consumo en la web: aunque a mitad de la temporada -marzo de 2018- el número de sus audios nativos era de 15 (los mismos que la Cadena SER), a partir de abril se irían añadiendo más propuestas (sobre música, cine, gastronomía y un serial de ficción) hasta elevar la cifra final a 21. Se evidencia así el objetivo de este operador de consolidar su transformación digital y convertir la web en una referencia informativa y de entretenimiento con contenidos originales de video y audio.

De los 21 podcast analizados en la investigación, COPE dedica once al deporte, lo cual revela, el aprovechamiento del conocimiento y capacidad de su plantilla para atender al aficionado a competiciones no atendidas en la parrilla (fútbol femenino, balonmano, tenis, fútbol sala). Sin embargo, y desde un punto de vista creativo, la mayor parte de estos programas se limita a replicar las estructuras productivas de la antena convencional: magazines con la participación de varios colaboradores que comentan y opinan sobre la actualidad de los respectivos deportes. Sin perjuicio del interés para los aficionados, la aportación radiofónica se valora limitada, poco innovadora y muy ligada a las lógicas analógicas, lo que no impide la eficiencia temática ni el potencial publicitario que brindan.

Esa inercia queda compensada por uno de los últimos estrenos de la temporada, el serial de ficción Sin Mi Identidad, que con guion, producción y dirección de Andoni Orrantia -subdirector de programación en COPE- evidencia de forma ejemplar el enorme potencial de la radio como creador de productos sonoros diferenciales y adaptados a las narrativas del nuevo entorno desde historias que se aproximan a la actualidad con relatos sugerentes y atractivos para públicos no atendidos en antena.

Tabla 4: Oferta de podcasts nativos en la Cadena COPE

\begin{tabular}{|l|c|c|c|c|}
\hline \multicolumn{1}{|c|}{ Título } & Tema & Subtema & Duración & Periodicidad \\
\hline This is Futbol & Deportes & Fútbol internacional & $1: 30^{\prime}$ & Semanal \\
\hline Showtime & Deportes & Baloncesto & $1: 05^{\prime}$ & Semanal \\
\hline Futsal COPE & Deportes & Fútbol sala & $1: 00^{\prime}$ & Semanal \\
\hline De Rosca & Deportes & Balonmano & $1: 00^{\prime}$ & Semanal \\
\hline El Albero & Cultura & Toros & $1: 00^{\prime}$ & Semanal \\
\hline Esto es fútbol & Deportes & Fútbol (inferiores) & $1: 09^{\prime}$ & Semanal \\
\hline COPE GP & Deportes & Otros (motor) & $1: 00^{\prime}$ & $10-15$ días \\
\hline Tercer Tiempo COPE & Deportes & Otros (rugby) & $1: 00^{\prime}$ & Semanal \\
\hline Fit Run COPE & Deportes & Otros (fitness) & $1: 00^{\prime}$ & Semanal \\
\hline COPEdaleando & Deportes & Otros (ciclismo) & $30^{\prime}$ & Discontinua \\
\hline Area Cbica COPE & Deportes & Fútbol (femenino) & $50^{\prime}$ & Semanal \\
\hline Planeta Eurosport & Deportes & Otros (miscelánea) & $1: 00^{\prime}$ & Semanal \\
\hline Hollywood land & Cultura & Cine & $8-^{\prime}$ & Semanal \\
\hline Geniales COPE & Educación & Otros (discapacidad) & $30^{\prime}$ & Quincenal \\
\hline Hablar en familia & Educación & Otros (familia) & $58^{\prime}$ & Semanal \\
\hline Tiempos modernos & Cultura & Música & $30^{\prime}$ & Semanal \\
\hline COPE y acción & Cultura & Cine & $8^{\prime}-9^{\prime}$ & Semanal \\
\hline De música ligera & Cultura & Música & $1: 00^{\prime}$ & Semanal \\
\hline Oído cocina & Cultura & Otros (gastronomía) & $1: 00^{\prime}$ & Quincenal \\
\hline De J. Carlos a Felipe & Cultura & Política & $14: 00^{\prime}$ & Única \\
\hline Sin mi Identidad & Ficción & Política & $14: 00^{\prime}$ & Semanal (8) \\
\hline Así fue el verano de & Cultura & Sociedad & $12^{\prime}$ & Semanal \\
\hline
\end{tabular}

Igualmente positiva se considera la aportación de COPE en el ámbito de la divulgación y la orientación pedagógica, que resulta apropiada por la adecuación de estos podcasts a la línea editorial del grupo y por la búsqueda de perfiles de audiencia más segmentados y poco atendidos en el resto de las ofertas. En este sentido, Hablar en familia, un programa semanal dedicado a los retos que supone la 
educación de los adolescentes para una generación de padres que hoy constituyen el grueso de la población actual en España (los adultos entre 40 y 60 años representan el baby boom nacido en nuestro país entre 1960 y 1975) se debe considerar no solo acertado en el contenido, sino estratégico en su alcance (según datos de la propia cadena, este podcast es uno de los más escuchados tras El Alberto y De Rosca). Como observación, se hace notar que la localización de los audios nativos en la web de COPE (bajo el epígrafe «solo en COPE.es») no resultó fácil de localizar ni cómoda de navegar, y que algunos de esos audios solo pudieron ser consumidos en streaming, sin descarga, lo cual dificultó su consumo en dispositivos móviles.

\subsection{ONDA CERO}

Entre los temas tratados en los podcasts nativos de Onda Cero predomina también el deporte, eje de la mitad de su oferta que atiende a modalidades como el baloncesto y el fútbol de segunda división y femenino. La música y los temas sociales se reparten el restante cincuenta por ciento. La primera es el motivo central de Rock and Roll Animal, un podcast pionero en la radio española porque surgió en 2008. Finalmente, los temas sociales -migración, diversidad cultural, desempleo- están presentes en Latitud Cero y Contraparte. Todos ellos son de producción propia: los realizan profesionales de la cadena que, además de afrontar sus respectivos programas en la antena, registran fuera de ella estos espacios sobre temas cuya trayectoria en la casa les convierte en especialistas.

Tabla 5: Oferta de podcasts nativos en Onda Cero

\begin{tabular}{|l|c|c|c|c|}
\hline \multicolumn{1}{|c|}{ Título } & Tema & Subtema & Duración & Periodicidad \\
\hline Juego de plata & Deportes & Fútbol & $35^{\prime}$ & Semanal \\
\hline Ellas juegan en la onda & Deportes & Fútbol & $35^{\prime}$ & Semanal \\
\hline Onda fútbol & Deportes & Fútbol & $35^{\prime}$ & Semanal \\
\hline 4 cuartos & Deportes & Baloncesto & $35^{\prime}$ & Semanal \\
\hline 10 historias 10 canciones & Cultura & Especiales (música) & $40^{\prime}$ & Semanal \\
\hline Rock and Roll animal & Cultura & Especiales (música) & $40^{\prime}$ & Semanal \\
\hline Latitud Cero & Sociedad & Otros (inf. internacional) & $25^{\prime}$ & $10-15$ días \\
\hline Contraparte & Sociedad & Otros (solidaridad) & $60-90$ & Semanal \\
\hline
\end{tabular}

En Onda Cero la línea continuista del podcast nativo respecto a la radio convencional se refleja en varios aspectos. Los programas mantienen en sus títulos la estrategia de la cadena de encabezar sus programas acudiendo a las palabras 'onda' o 'cero': Latitud Cero, Onda fútbol y Ellas juegan en la onda, en la línea de Julia en la Onda o Aquí en la onda, veteranos programas en emisión hertziana. Respecto a la periodicidad, la de la mayoría de los podcasts nativos son semanales, frecuencia también habitual en la radio tradicional. El seguimiento sobre las de descargas y usuarios únicos de estos programas le sirve a Atresmedia como indicador a partir del que decidir si se incorporan a su ventana analógica.

Aunque la cadena vería con buenos ojos el patrocinio de sus podcasts nativos, ninguno se comercializa bajo esta fórmula. Esto se debe, según Mónica Muñoz, responsable de contenidos web del grupo de comunicación, a la dificultad que entraña cuantificar su audiencia, para la que el Estudio General de Medios es insuficiente. Pese a que Onda Cero tiene sus propios sistemas de medición de descargas y suscripciones, a la hora de monetizar el producto la carencia de datos sólidos e irrebatibles disuade a los potenciales anunciantes.

\section{DISCUSIÓN Y CONCLUSIONES}

La consolidación de los hábitos de consumo digital basados en el irrenunciable paradigma any content, anywhere, anytime, any device (cualquier contenido ha de disfrutarse en cualquier momento, lugar y dispositivo) ha obligado a los operadores tradicionales de radio a ampliar sus repositorios sonoros en la web -hasta no hace mucho basados únicamente en lo emitido en antena- con productos exclusivos, 
diferenciales y competitivos. Con ello se intenta atraer a nuevos usuarios para multiplicar la base de oyentes, reforzar la identidad de cada medio y conseguir vías de comercialización complementarias a las propias del negocio hertziano. La investigación deja probada la hipótesis de que la producción de podcasts nativos en la radio comercial española, que sí ayuda a posicionar las respectivas marcas en el entorno online al atraer al usuario a sus repositorios digitales, prioriza aún la cantidad sobre la calidad, pues ni los contenidos ni los tratamientos narrativos observados en la mayoría de los audios creados para su difusión exclusiva por este canal suponen una aportación relevante o alternativa frente a la programación convencional. No obstante, resulta necesario matizar esta afirmación atendiendo a los siguientes indicadores:

\subsection{TEMAS Y DURACIÓN}

Aunque se constata que el abanico de contenidos abordados por el conjunto de radios generalistas comerciales intenta renovar el repertorio de temas y públicos que dominan las parrillas (información, deporte y entretenimiento, salvo en los fines de semana), la mayoría de los podcasts nativos (la mitad del total) se dedican al deporte. Más aún, los más antiguos tanto en la Cadena SER como en COPE profundizan sobre la actualidad de la jornada del fin de semana (This is fútbol, Play fútbol), estrategia que se demuestra más dirigida a la fidelización de los oyentes de la emisión convencional que a la búsqueda de nuevos seguidores.

Con todo, hay bastantes podcasts nativos deportivos que atienden a especialidades poco habituales en la radio hertziana: baloncesto, balonmano, fútbol femenino, de categorías inferiores, ciclismo, motor, tenis, running... Es aquí donde el formato de audio a la carta permite completar la oferta deportiva de las cadenas comerciales y exprimir el rendimiento de sus redactores, a veces con una trayectoria y reconocimiento personal que ayudan a posicionar mejor este producto digital (por ejemplo, el especialista en balonmano de COPE Luis Malvar en De Rosca).

Con respecto al resto de áreas temáticas, merecen destacarse las apuestas divulgativas de COPE (Hablar en familia y Geniales COPE), muy en línea con su declaración de identidad, así como las propuestas digitales de Onda Cero, donde se enfatiza más el valor diferencial de los conductores (Carlos Alsina y sus colaboradores, Manuel Marlasca o Guillermo Fesser) que el de los propios contenidos que se abordan en cada caso.

En cuanto a la duración, y aunque se constata gran heterogeneidad en el conjunto y hasta en las propias cadenas, se diría que las lógicas de la radio analógica aún se imponen a la hora de concebir y producir los podcasts nativos: solo 2 de los 44 duran menos de 10 minutos, lo cual parece contradictorio con las demandas de consumo acelerado y casual que se aprecian en el entorno digital (situaciones de tránsito, consulta puntual, experiencia rápida y eficiente). Dominan los programas con duración cercana a una hora, casi siempre de producción semanal, que revela una mentalidad de los creadores más anclada a los sistemas de trabajo de la radio hertziana que a los del nuevo ecosistema: este parece otro de los retos más acuciantes para dotar a este producto de personalidad propia y diferencial junto con una frecuencia elevada en la discontinuidad de la producción de contenidos online.

\subsection{Publicidad}

La encrucijada que todavía condiciona a los operadores con respecto a los podcasts nativos es que los anunciantes digitales puedan restar ingresos a las ondas, y por ello se aprecia cómo la búsqueda de la rentabilidad web está supeditada a la conservación de las fórmulas propias de la emisión analógica. Ninguna de las tres cadenas potencia la monetización de los contenidos digitales, y prueba de ello es que, en la mayoría de los casos son los propios redactores quienes producen y desarrollan los podcasts nativos (sobre todo deportivos y culturales) sin una estrategia comercial definida, se diría que más por afición o inquietud personal.

Como ya se ha apuntado, esta dinámica afecta a los temas objeto de podcast nativos, en su mayoría deportivos: semejante segmentación permite abrir los espacios publicitarios a anunciantes y productos

(C) Ediciones Universidad de Salamanca / CC BY - NC ND Fonseca, Journal of Communication, n. 17, 2018, pp. 91-106 
mucho más orientados al público aficionado a cada especialidad (por ejemplo, el running o el rugby), pero todavía no se incluye ningún ejemplo en este sentido. Aquí se identifica otro de los grandes objetivos por lograr en las apuestas de audio digital de la radio.

\subsection{GÉNEROS}

Este indicador también revela que el podcast nativo aún está en una fase incipiente de desarrollo en la radio: lo demuestra el uso de lenguajes y géneros que en nada se diferencian de la radio tradicional y que, como esta, recurre con frecuencia al magazine como género, es decir, al comodín que ha conformado, por tradición, uno de los bloques de programación más explotados en la mañana o por la tarde. Dicho de otro modo, están por explotar las posibilidades de un medio diferente a la radio: el podcast no debe concebirse, salvo excepciones, como una «radio en diferido», fragmentada con mayor o menor acierto y distribuida de un modo más o menos cómodo para que el usuario acceda a ella. Estamos a cierta distancia de concebir el podcast como «una fórmula nueva de distribución en el sentido más amplio de la palabra: es radio, pero bajo nuevas reglas puesto que la relación con el oyente cambia por completo» (Gallego, 2010, p. 261). El podcast como variante del medio radio está por consolidarse.

Bien es cierto que en este proceso también se descubren necesarias algunas reglas aún no asentadas en la conceptualización y distribución de este formato: algunos de los podcast más descargados en el audiokiosko iVoox difícilmente pueden ser calificados como tales, pues su único parecido con el audio es que son sonoros: documentales de cadenas como Discovery Channel (Documentales sonoros, el quinto programa más descargado de iVoox en septiembre de 2018), o shows televisivos como El chiringuito de jugones (presentado en el canal de Atresmedia Mega por Josep Pedrerol, alcanzó el puesto 31 en el mismo listado). Quizá el concepto podcast resulte hoy demasiado ecléctico y ello no permite asociarlo aún a un lenguaje y una naturaleza propios.

\subsection{RENOVACIÓN DE CONTENIDOS}

La mayoría de los podcasts nativos de la radio generalista española, más del 70\%, proviene de años anteriores, lo que, por un lado, constituye un indicador de su aceptación y de la aceptación del formato. No obstante, el podcast no está sujeto temporalmente a la configuración de una parrilla estándar: a lo largo de la temporada analizada nuevos podcasts se incorporan a la oferta a medida que esta avanza, mientras que los que dejan de producirse permanecen al alcance del usuario, siendo en algunos casos más exitosos en cuanto a número de descargas que los que continúan elaborándose. Es el caso de Historias del valle sin retorno, de Guillermo Fesser, que dejó de producirse en 2016 y cuyos 52 capítulos siguen formando parte de la oferta nativa de Onda Cero dado su elevado número de descargas.

Entre los podcasts que se incorporaron durante la presente temporada cabe reseñar, por ejemplo, Oído cocina, un espacio gastronómico presentado por Urbano Canal y Roberto Pablo en COPE. Este programa llegó a la oferta de podcasts nativos de la cadena en abril de 2018, es decir, a mitad de temporada, y su buena acogida sirvió para que se convirtiese en un programa de la antena convencional desde el mes de julio: una prueba de que el entorno digital se empieza a contemplar como escenario para el testeo y la experimentación de cara al, todavía, preferente negocio de la antena.

No obstante, los crecientes indicadores sobre el consumo de audio digital y el previsible impulso que recibirán con la llegada al mercado de los altavoces inteligentes -dispositivos que ofrecen únicamente contenidos de vOz- permiten aventurar una apuesta más decidida de los operadores por el podcast nativo, el mejor exponente de la transformación digital de la radio y de su pervivencia a medio y largo plazo.

\section{BibLIOGRAFÍA}

Anderson, C. (2004). The Long Tail. Wired Magazine, 12 (10), pp. 170-177. https://doi.org/10.3359/oz0912041 
Balsebre, A., Ricarte, J. M., Perona, J. J., Roca, D., Barbeito, M. L., y Fajula, A. (2006). Los mitos de la publicidad radiofónica. Madrid: Cátedra.

Berry, R. (2006a). Will the iPod kill the radio star?: Profiling podcasting as radio. Convergence, 12(2), pp. 143-162. https://doi.org/10.1177/1354856506066522

Bonini, T. (2015). The 'Second Age' of Podcasting: reframing Podcasting as a New Digital Mass Medium. Quaderns del CAC, 18(41), pp. 21-30. Recuperado a partir de http://www.cac.cat/pfw files/cma/recerca/quaderns_cac/Q41_Bonini_EN.pdf

Casas Santasusana, E. (1954). Publicidad por radio. Barcelona: Juan Bruguer.

Catalina-García, B., López de Ayala, M. C. y Martín Nieto, R. (2017). Audiencia juvenil radiofónica: uso de nuevos dispositivos y aplicaciones entre los futuros comunicadores. Revista de la Asociación Española de Investigación de la Comunicación, 4(8), pp. 45-53.

Díaz Nosty, B. (Coord.) (2017). Diez años que cambiaron los medios (2007-2017). Barcelona: Ariel y Fundación Telefónica. Recuperado de https://www.fundaciontelefonica.com/arte cultura/publicaciones-listado/pagina-itempublicaciones/itempubli/602/

European Broadcasting Union. (2017). Trust in media. Recuperado de https://www.urp.ch/publications/trust-in-media-2017

Franquet, R. (2017). Un medio versátil en los albores de su centenario. Diez años que cambiaron los medios (20072017). Barcelona: Ariel y Fundación Telefónica (pp. 212-219).

Gallego, J. I. (2010). Podcasting, nuevos modelos de distribución para los contenidos sonoros. Barcelona: UOC.

Hammersley, B. (2004). Audible Revolution. The Guardian. Recuperado de https://web.archive.org/web/20130922040919/http://www.theguardian.com/media/2004/feb/12 broadcasting.digitalmedia

Jobs, S. (2001). 2001 Apple Music Event. Recuperado de https://www.youtube.com/watch?v=kNOSVBCJqLs

La SER se queda sin «Carne Cruda». (2014, julio 9). Eldiario.es. Recuperado a partir de https://www.eldiario.es/cultura/fenomenos/queda-Carne-Cruda 0 279622410.html

López Vidales, N., Gómez Rubio, L. y Redondo, M. (2014). La radio de las nuevas generaciones de jóvenes españoles: hacia un consumo online de música y entretenimiento. ZER, 19(37), pp. 45-64.

Moreno, L. (2017). Podium Podcast, cuando el podcasting tiene acento español. Prisma Social, 18. Recuperado a partir de http://revistaprismasocial.es/article/view/1418

Morla, J. (2016, junio 9). Nace Podium Podcast, un nuevo modo de escuchar. El País. Recuperado de https://elpais.com/cultura/2016/06/08/actualidad/1465410435 296991.html

Muñoz, M. (2018). Entrevista personal con la responsable de contenidos web de Atresmedia.

Nafría, I. (2008). Web 2.0: El usuario, el nuevo rey de Internet. Madrid: Gestión 2000.

Orrantia, A. (2018). La triple secuencia multiplataforma y la audiencia social en COPE. La narración distribuida de contenidos en la antena, la web, redes sociales y alertas a teléfono móvil durante el Día de la Radio en 'Herrera en COPE'. Hipertext.net, 16, mayo, 43-50.

Pedrero Esteban, L. M. y Pérez Alaejos, M. (2018). La transición de la radio española al entorno digital: experiencias y retos de las estrategias transmedia. En E. Galán, E., A. Rodríguez y J. Marzal (eds.), Contenidos transmedia para la radiotelevisión de proximidad (pp. 321-333). Pamplona: Eunsa.

Pedrero Esteban, L. M. (2000). La radio musical en España, historia y análisis. Madrid: IORTV.

Pérez Alaejos, M. de la P. y López Merayo, A. (2013). Entre ondas y bits el podcasting en las redes nacionales de radio españolas. Comunicação Midiática, 8(3), pp. 181-202.

Rodríguez Pallarés, M. (2016). Reutilización de la ficción sonora en la Cadena SER. El caso de Podium Podcast. Área Abierta. Revista de Comunicación Audiovisual y Publicitaria., 17(1), pp. 83-97.

Siemens, G. (2008). Learning and knowing in Networks: Changing Roles for educators and designers. ITFORUM for discussion. Recuperado de: http://itforum.coe.uga.edu/Paper105/Siemens.pdf 
Sociedad General de Autores y Editores. (2017). Anuario SGAE de las artes escénicas, musicales y audiovisuales. Recuperado de http://www.anuariossgae.com/anuario2017/home.html

Tenorio, I. (2008). La nueva radio. Barcelona: Marcombo.

Van Zanten, R., Somogyi, S. y Curro, G. (2012). Purpose and preference in educational podcasting. British Journal of Educational Technology. https://doi.org/10.1111/j.1467-8535.2010.01153

Videla-Rodríguez, J. J. y Piñeiro-Otero, T. (2013). La radio móvil en España. Tendencias actuales en las apps para dispositivos móviles. Palabra Clave, 16(1), pp. 129-153.

Witt, S. (2016). Cómo dejamos de pagar por la música. Barcelona: Contra. 